\title{
Toward an Autonomous Incubation System for Monitoring Premature Infants
}

\author{
Hameed AIQAHERI ${ }^{1}$, R. SUJATHA ${ }^{2 *}$, Jyotir Moy CHATTERJEE ${ }^{3}$, \\ Sridharan SHOORIYA ${ }^{2}$, Sai Aswin KUMAR J², Neha SATISH ${ }^{2}$ \\ ${ }^{1}$ College of Business Administration, Kuwait University, Kuwait \\ alqaheri@yahoo.com \\ ${ }^{2}$ SITE, Vellore Institute of Technology, Vellore, India \\ r.sujatha@vit.ac.in (*Corresponding author), sridharan.shooriya2017@vitstudent.ac.in, \\ saiaswin.kumarj2017@vitstudent.ac.in,neha.satish2017@vitstudent.ac.in \\ ${ }^{3}$ Department of IT, LBEF \& Scientific Research Group in Egypt, Kathmandu, 44600, Nepal \\ jyotirchatterjee@gmail.com
}

\begin{abstract}
The incubator is an encased device whose inward ecological system is disconnected from the surrounding climate. It creates an ideal climate for the incubation process and is an indispensable device for premature infants as these children are prone to developing health problems that would affect the conditions of their growth and development. A low-cost, IoT-based autonomous Incubation System that would create an ideal environment for premature children to help them to grow to maturity would be invaluable, especially for rural areas that lack adequate medical facilities. Such a system would significantly reduce the mortality rate of premature infants. This paper focuses on designing a framework for such a system in which the temperature and humidity are autonomously controlled. A secured incubation system was developed using IBM Cloud, IBM Watson IoT platform, and MIT App Inventor.
\end{abstract}

Keywords: Incubator, IoT, Premature infants, Cyber security, Cyber-attack prevention.

\section{Introduction}

Preterm infant care is one of the most significant, representative, and sensitive areas in the clinical field. Some newborns, called high-risk infants, are at more severe risk of mortality because of their gestational age, and their introduction into the world weight causes life-threatening illness and even demise. Premature children need to be protected in an environment comparable to that of the uterus in order to accommodate external conditions. To some extent, the incubator fulfils his purpose. Baby incubators can provide stable temperature and relative humidity to a degree in which the preterm has the same condition as in the womb. Air temperature and relative dampness must be maintained as required by preterm infants. Estimations of these limitations are possible, and under severe circumstances, this innovation framework should be passed with caution on to the parents. Arduino and IBM clouds were utilized for executing such equipment.

Because data represent the most valuable resource, the system must protect its confidentiality, integrity, and availability. Therefore, many security checks must be performed to prevent intrusion on the website.

Rural areas lack the medical facilities or offices required to support an electrical gadget like an incubator and are not sophisticated enough to be given for use to premature babies or those babies in need of the incubators. In addition, villagers who live there cannot bear the cost of such incubators without government support. Moreover, even if the government provides incubators or medical facilities to these hospitals, maintenance is not possible or available. Hence, infants die rapidly. Therefore, the goal is to create a low-cost, low-maintenance system that would help premature children by surrounding them with an ideal atmosphere for their growth while also monitoring their heartbeat, body temperature, and humidity. Furthermore, this system would also be helpful for the parents of newborns who are occupied with their everyday work. The Internet of Things (IoT) is the association of gadgets to the web. Industrial IoT (IIoT) stretches out IoT to incorporate help for modern applications like prescient upkeep. The gadgets that are being observed for industrial applications can incorporate conventional IoT gadgets associated with hardware or installed in areas. Here a strategy for programmed recognition of preterm infant's temperature and humidity in incubator and in open bed is proposed. Many infants from rural areas can be helped and saved simultaneously over a short period. 
The main contributions of this work are:

- The development of an autonomous or semiautonomous feasible low-cost, IoT-based infant incubator for the rural areas devices that control the temperature and humidity from the incubator with a mobile app, and that can also alert the doctor(s) in charge if there are any out of range changes in both the temperature and humidity values.

- The temperature and humidity are automatically set to the optimum values when they exceed the acceptable ranges;

- The device control would be hosted on the Internet so as to establish security control and security mechanism measures;

- Humidity and temperature recommended by pediatricians can be remotely monitored through the web or Android application captured by humidity and temperature sensors;

- Incubation prevents the spread of viruses and diseases in infants and helps infants grow healthy;

- The portal responsible for controlling IoT-based incubators is resistant and resilient to attacks.

The rest of the paper is organized as follows: Section 2 contains a literature survey of 12 similar papers condensed into a few words. Section 3 presents the proposed system. Section 4 includes the implementation details and steps and the results of the proposed system. Section 5 offers a discussion regarding the observed advantages and disadvantages of the present approach. Section 6 provides the conclusion of the proposed work and possible future work.

\section{Related Work}

Shabeeb et al. (2020) presented a system where the temperature and humidity levels in the newborn incubator were remotely checked remotely using an Arduino microcontroller with various sensors and open-source IoT applications. Their framework uses Arduino microcontrollers, DHT11/DHT22 sensors to estimate body conditions, such as temperature and humidity, LCD screen, ESP8266 WIFI module, and NodeMCU-v3. The results showed that the ThingSpeak IoT application can continuously transfer clinical records to clinical staff.
Ishak et al. (2017) presented a framework for examining newborns in an incubator and recording important information in a PC. For indicative or inspection purposes, neonatal emergency room staff may provide further information on the framework records. The survey revolves around building observation frames, including an incubator equipped with wet sensors to quantify heartbeat sensors and moisture.

In a human service-observing framework, numerous asset-constrained sensors are sent to detect, process, and impart data. Nonetheless, these ceaseless gadgets and exact tasks are significant, particularly in the newborn child hatchery observing framework. Since meaningful choices are made on the obtained data, it is crucial to guarantee the credibility of the hatchery checking framework and of specialists. In this work, an open key encryption-based computationally proficient shared confirmation convention is proposed for secure information transmission between hatchery observing frameworks and specialists or managers. The proposed convention improves execution and reduces computational expense without trading off security (Jegadeesan et al., 2019).

As stated by Koli et al. (2018), any temperature variation can influence all living beings and semiconductor materials. Their venture is to control the adjustments in temperature for specific applications, such as child incubators. The overall hatchery is utilized for newborn children to improve infants' endurance by giving them warm conditions and lessening the lack of warmth from the infant's body. Fundamentally, the incubator is the standard strategy used worldwide. The present approach utilizes Arduino, a temperature sensor for checking or controlling a child's body temperature. The steady temperature is between $36.5-37.2^{\circ} \mathrm{C}$ as the child requires when being in his mother's womb.

Weber et al. (2021) proposed a strategy for programmed recognition of a preterm infant's presence in incubator and open bed.

The control framework utilized in (Rakhmawati et al., 2019) poultry incubation utilizes ON/OFF control and is checked with a local area network (LAN). A control framework utilizing ON/OFF control produces precarious warming. Accordingly, this examination presents the advancement of the 
egg incubator framework utilizing fuzzy approach and is dependent on IoT. Thus, the egg brings forth the framework which is steadier when it heats the temperature in the incubator and can be controlled through the web arrangement.

Latif et al. (2021) meant to plan a temperature identifier for a baby incubator that had a steady temperature and was checked straightforwardly in a staff room.

In the study of Wang, Peng \& Gu (2017) a planned review of the existing IoT-based sensors and a sensor framework from the IoT market value were systematically described. Comparative investigations of the sensors have been done. Ray et al. (2020) proposed security and protection issues related to sensor information and ways to address these issues. For instance, a baby incubator was used to present the employment of IoT innovation in order to lessen the danger involved by the utilization of clinical gadgets. The administration level and productivity were improved through a dynamic administration.

Rajalakshmi et al. (2019) focused on accessible incubator monitoring frameworks, estimated organic boundaries and examinations procedures achieved by utilizing continuous observation and information transmission. They planned to structure an android-based newborn child incubator that could be obtained and controlled utilizing the Android application over the Internet. The structured framework (Inam et al., 2019) can gather the incubator's condition and store it on an online worker. The framework could assume an impressive job in decreasing the death rate of untimely newborn children in far-off territories.

Tej et al. (2021) executed and presented a viable medical services perception and checking system using RFID labels and IoT.

The framework proposed by Sheet et al. (2019) comprised of Arduino microcontroller (Atmega), sensors for measuring the humidity, the temperature, the percentage of $\mathrm{CO} 2$ and the water supply level. Moreover, this framework was an actuator utilized as an on/off turning control for the fan and the heater.

Ashish (2017) introduced an expense commendable plan of a gadget for a constant monitoring of the infants in the incubator, which allows early recognition of potentially dangerous occasions and keeps a protected climate for the newborn.

Azamir et al. (2021) focused on improving the humidity and temperature checking frameworks on egg incubator machines, particularly on those that required a constant observation.

A neonatal incubator fused with implanted temperature and humidity control framework has been proposed and created which is Nisha \& Elahi (2014).

\section{Proposed Work}

The proposed autonomous Incubation System for monitoring premature infants regulates temperature and humidity to help premature infants to grow up and evolve. Such a system would lead to an increase in the child-living ratio worldwide. Because the evaporative loss could reach up to $20 \%$ in the case of a newborn infant, the temperature and humidity must be modified in a controlled manner to prevent the infants' loss of weight (Abdiche et al., 1998). The ideal temperature and humidity for premature babies to grow in an incubator are between 96 and 98 degrees Fahrenheit and between 60 and 77 percent, respectively. These parameters play an important role in the proposed system, which contains temperature and humidity sensors connected to the Arduino board. These are temperature and humidity controllers employed to control the ideal temperature and humidity of premature infants. Many studies have proved the ideal temperature and pressure, the same values are used for the incubator system proposed in the paper. The novelty of the proposed system is that it is autonomous and hosted over the Internet. Hence, doctors living even in a remote corner of the world with access to the Internet connection could control the incubator and be aware of the condition of the premature babies in the incubators. The data collected by the incubator are stored securely for future analysis and finetuning of the system.

The infant's temperature and humidity were closely monitored using temperature and humidity sensors, respectively.

The incubator device is securely hosted on a web server and the devices are remotely controlled 
over the Internet. Secure web-based applications to detect and prevent intrusions or attacks with IP address logging, and other security mechanisms are built. The website that hosts the device is resistant and resilient to attacks. Therefore, the website that controls the IoT-based incubator is carefully coded with state-of-the-art secure coding mechanisms and well-proven practices. Various cyber-attacks on IoT devices have been noted, and the portal has been made to prevent security intrusions.

The proposed architecture of an autonomous infant incubator is illustrated in Figure 1. It depicts the working conditions and functionality of the system. First, the system gathers information about the infant, sets the optimal conditions based on that, and displays the LCD. IoT devices can be controlled via web applications and Android devices.

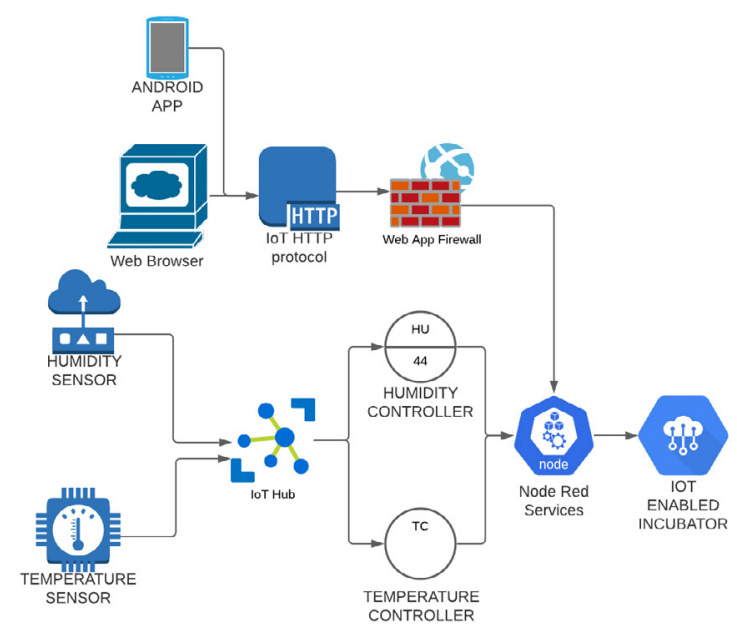

Figure 1. Proposed system architecture

The web interface architecture depicted in Figure 2 consists of web pages made up of the Document Object Model (DOM). JavaScript (JS) is responsible for manipulating the DOM. JS is also responsible for dynamic viewing and front-end validation. The radio resource control (RRC) takes place between the browser and the webserver. The browser sends the data after being validated, and on the server, the data are computed and stored in the database, which resides on the SQL server. Structured query language (SQL) is the communication medium between the web server and the SQL server. After communicating with the SQL server, the web server returns the result to the browser via the RRC.
This section presents 2 modules (IBM Cloud Module and IBM IoT Module) and 10 submodules (IBM IoT Platform, IBM IoT simulator, NODERED module, WEB APP module, MIT APP INVENTOR module, UI module, IP address and user details logging module, DOS attack prevention module, ADMIN module (Web Interface) and Register module).
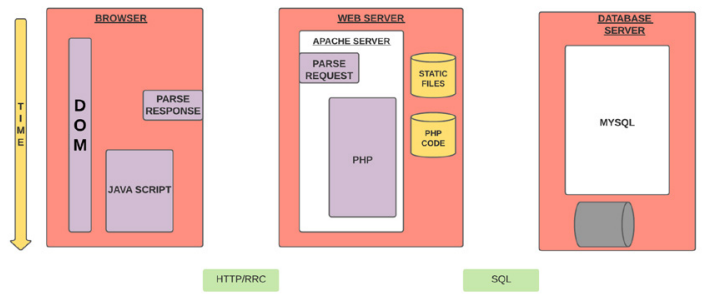

Figure 2. Web interface architecture

\section{IBM Cloud Module}

IBM Cloud provides cloud management for business infrastructure as a service (IaaS), software as a service (SaaS), and platform as a service (PaaS) through a private, public, community, and hybrid cloud delivery model.

\section{IBM IoT Module}

The IBM IIoT gives a way of displaying the gadget progressive system that is incorporated as a component of an answer and of obtaining data from those gadgets by utilizing industrystandard conventions.

- IBM IoT Platform: IBM Watson IoT Platform ingests gadget information, changes that information into meaningful experiences, streamline-cycles, and guides new item plans. It captures data in real-time and optimizes operations and resources to increase revenue. In this module, the primary device for the system was created and added. Temperature and humidity sensors were set up to calculate the infant data. With varying temperature and humidity of infants, the parameters were corrected to ensure the success of the incubation operation. The infant's temperature and humidity were set automatically to an optimum value, and the parameters were corrected automatically. The sensors were connected to the added device and inputs were sent through a Python program;

- IBM IoT simulator : The essential instrument that gadgets and applications use to speak 
with the IBM Watson IoT stage is MQTT. This convention is intended for the skilled trade of ongoing information with sensors and cell phones. The IBM IoT simulator has virtual sensors connected with the IBM IoT platform, thus performing an IoT simulation;

- NODE-RED module: Node-RED is a stream-based progression device for IBM $>\mathrm{s}$ visual programming for wiring hardware devices, APIs, and online organizations as a part of the IoT. Different nodes were created and configured in node-red for input and output, respectively. It also provides support for networking nodes separately for linking with non-IBM services;

- WEB APP module: A web app is created using the node-red module. It has a user interface to show the current values of the temperature and humidity sensors in the gauges. The optimum values of these two parameters were set automatically based on the infantss condition;

- MIT APP INVENTOR module: This module is an extension of the nodered module and web app module. MIT App Inventor is a web application that incorporates a development environment for programmers created by Google to make Android applications. This app contains a user interface with labels (temperature and humidity), text fields for temperature and humidity, and buttons to control the app;

- UI module: This module was built using HTML, CSS, JS, Bootstrap framework, and WordPress. The dynamic viewing of content on changing screen sizes was built using built-in CSS classes in bootstrapping. The entire user interface and front end consist in this module;

- IP address and user details logging module: When a particular IP address makes a request, it is logged in the database along with other information such as status code, country code, country name, region name, zip code, latitude, longitude, time zone, and the time the details have been stored. This was implemented using an API called IPInfoDB, which automatically collects this information. Details have been stored in DB using MYSQL, PHP, and JSON. All details are stored dynamically for every request. The two tables store the IP details.
The first table stores all the visiting IPs. It is insensitive to being duplicated. The second table stores the non-duplicated IP addresses. If a new IP enters or makes a request, the IP details are stored;

- DOS attack prevention module: This module is embedded on the landing page of the website. In the worst-case scenario, the denial of service crashes the website. This is a compulsory module that has been developed. This module was developed using PHP and MySQL. Because all the landed IP addresses are stored in the database with a timestamp, this can prevent DOS by checking if the current IP had more than 100 requests in the past one minute. Then, it temporarily blocks the incoming requests from a particular IP address, thereby preventing DOS and DDOS attacks;

- ADMIN module (Web Interface): This is the core module of the proposed idea. It has many coupled submodules. It has low coupling and high cohesion to enhance scalability;

- Register module: The user can enter details in the registration form. The details were appropriately validated. The user inputs were adequately cleaned before being added to the database table.

Then, a confirmation email is sent to the owner. The owner clicks the verified link and activates the account; otherwise, the account remains inactive. This prevents normal users without admin privilege from creating accounts and bypass access controls. This is an important security check that has been implemented. The passwords are hashed using the MD5 (Message Digest 5) algorithm before storing them in the table. In the worst-case scenario, it will be difficult for the attacker breaking the system to find the password because of the time issue being mitigated.

\section{Implementation and Results}

The used hardware components were: 1) Arduino; 2) the temperature sensor; 3 ) the humidity sensor; 4) the LCD and 5) the connecting wires. The used software components were: 1) the IBM cloud; 2. the IBM IoT platform; 3) the IBM Watson; 4) the Node-red; 5) the Tinker CAD; 6) the Python IDLE and 7) the MIT app inventor. The stepwise 


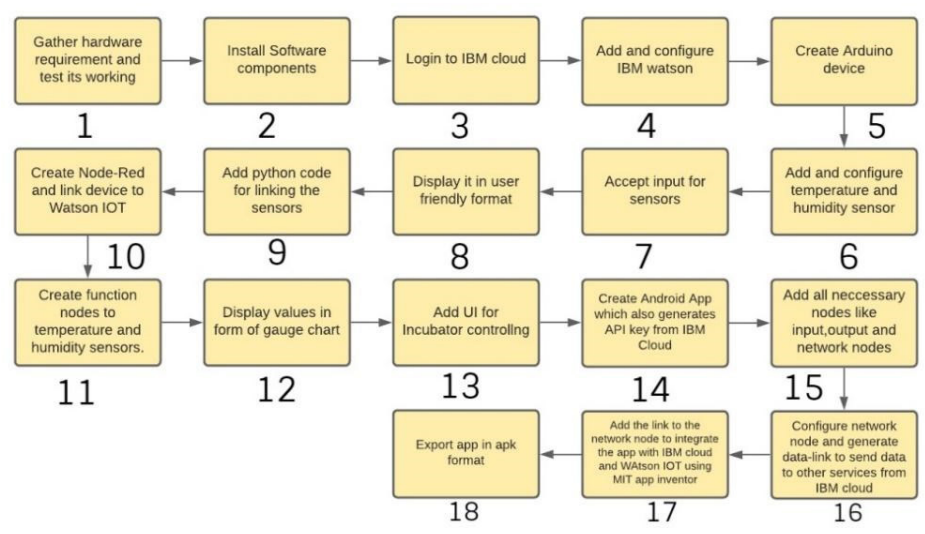

Figure 3. Working of the proposed system

operation of the proposed system is shown in Figure 3.

Cumulatively the implementation process could be summarized as follows:

- The first step is to gather all required hardware components and test their working;

- The next step is to install all the software components for a proper functioning of the system;

- Create or log into IBM cloud account;

- Add and configure IBM Watson IoT from IBM cloud;

- In IBM Watson IoT create a new device (here Arduino) to start the IoT system;

- Configure the created device with primary details asked and store the data safely and confidentially;

- Go to IBM sensor simulator website and add and configure the required sensors (here temperature and humidity sensors) with the device data that were stored (from the previous step) and start to link the sensors with the device in the IBM Watson IoT, in the IBM cloud;

- In the IBM Watson IoT platform, create charts (graphs) for the sensors to accept the input and output from the sensors, in the same user-friendly format;

- Create python code and link it with the sensors to send input to the sensors in order to set temperature and humidity respectively;
- Create node-red (JSON) and link the device created in the IBM Watson IoT using "IoT IN" node by providing the stored details;

- Create two functional nodes to add functions to temperature and humidity sensors to create a user interface and start a web app;

- Create the output format like a gauge, a chart or a bar graph to depict the sensor values (here, a gauge is used);

- Add buttons to the UI to control incubator system using the web app;

- To create an android app first generate an API key from the IBM cloud;

- Head over to MIT app inventor website and $\log$ in/sign up;

- $\quad$ Select create new app and enter the API key generated from the IBM cloud to synchronize with the IBM cloud and Watson IoT to link with the device;

- First select the frontend button to design the UI for the app;

- Here, textboxes, labels and buttons were used.

- After completing the above step, head over to the backend by pressing the backend button in the MIT app inventor;

- Add all the necessary nodes like input nodes, network nodes and output nodes;

- In node-red, add and configure network node and generate network data link to send data from IBM cloud to other services; 
- In MIT app inventor, add the link to the network node to integrate the app with the IBM cloud Watson IoT;

- Finally export the app in .APK format to share the app with the users or provide QRcode to download the app directly from MIT app inventor.

\section{Android App}

The Android app is made using MIT App Inventor (originally developed by Google and currently maintained by MIT). It is connected to the NodeRed Platform to obtain input for the temperature and humidity placeholders to the user.

The android application depicted in Figure 4 can switch on or off the incubator and set the ideal temperature and humidity for premature infants, having some special conditions as presented in Figure 5, and shows that the humidity was maintained between $60 \%$ and $77 \%$.

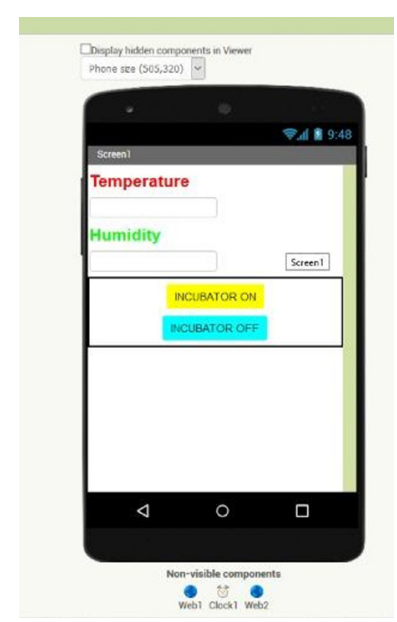

Figure 4. Android App

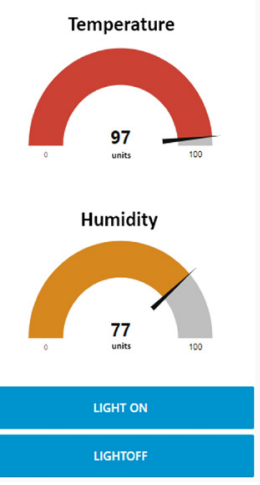

Figure 5. Sensor Values

The most common web penetration attacks, such as Brute-forcing passwords, CSRF, Distributed Denial of Service Attacks, SQL injection, HTML injection, Cross-Site Scripting (XSS), and Insecure Direct Object Reference (IDOR) are prevented. All IoTbased devices are created in the IBM IoT platform, and every action and integration occurs there.

Node-Red is a JSON-based flow action platform used to connect IoT-based devices to a userunderstandable format. That is, it is linked to the creation of UI and apps.

The web app was created using the NodeRed Platform. It has a UI that represents the temperature and humidity output values and allows user controllability. The values of the humidity and temperature are graphically illustrated in Figure 5 in a meter chart format.

\section{Temperature Sensor}

The TMP36 sensor is a simple way of estimating temperature utilizing an Arduino. The sensor can gauge an extended temperature range (from $50^{\circ} \mathrm{C}$ to $125^{\circ} \mathrm{C}$ ), is genuinely accurate in measurements $\left(0.1^{\circ} \mathrm{C}\right.$ resolution), and involves extremely minimal expense, well-known decision being settled on it.

\section{Humidity Sensor}

The DHT11 estimates relative humidity. The relative humidity is the measure of water fume in air versus the immersion point of water fume in air. At the immersion point, water fume begins to consolidate and gathers on surfaces framing dew. The DHT11 recognizes water fume by estimating the electrical obstruction between two electrodes. The humidity detecting part is a dampness holding substrate with electrodes applied to the surface. DHT11 can gauge temperature from $0^{\circ} \mathrm{C}$ to $50^{\circ} \mathrm{C}$ with $\pm 2.0^{\circ} \mathrm{C}$ accuracy, and humidity from 20 to $80 \%$ with $5 \%$ accuracy.

\section{Admin Registration with Dynamic Form Validation}

Upon validating the form, the account is created and activated if the owner verifies it via email. The admin user will not be activated without an activation made by the owner. The security feature is implemented so that not all the users can create accounts. Only the authenticated users can create them. 


\section{Login}

The login has a security feature that prevents the brute-forcing of the password. If the password has been entered incorrectly more than five times, the account is blocked. It will be unblocked only if the 'forgot password' is clicked. Then, the new password is changed. This mechanism also prevents CSRF attacks. In this method, a password is changed without the user realizing it.

\section{Denial of Service Attack}

If $100 \mathrm{GET} / \mathrm{POST}$ requests come from a single user within a minute, the IP is blocked, and the connection is dropped. This makes the attacker unable to access the website or blocks him by loading requests on the website.

\section{Email Confirmation for Password Change Request}

When trying to change a password an email is sent to confirm if the user is the one who requested it, thereby enhancing security.

\section{Confirmation of Admin}

Admin accounts can only be created if the owner verifies them. Therefore, an email is sent to the owner's inbox.

\section{Admin's Control Panel}

This is the control panel in which the admin can manually block or unblock IP addresses. If the Admin blocks a particular IP, then the IP holder cannot visit the website, and his connection would automatically be dropped. The status of a particular IP was also viewed.

\section{SQL in Injection Prevention}

SQL injection can be prevented through a secure PDO package using placeholders rather than directly concatenating the SQL statements. HTML injection can also be prevented using the HTML entities() function in PHP.

The graph depicted in Figure 6 represents the real-time values of the temperature inside an IoTbased incubator. The $\mathrm{x}$-axis represents the time in minutes, and the $y$-axis represents the temperature plotted in Fahrenheit. The graph shows that the temperature varies from $96^{\circ}$ to $98^{\circ} \mathrm{Fahrenheit}$, which is the ideal temperature for premature infants to mature. This is briefly explained in the description of proposed system in Section 3.

The graph depicted in Figure 7 represents the real-time value of humidity in percentage inside an IoT-based incubator. The $\mathrm{x}$-axis represents the time in minutes, and the y-axis represents the plotted humidity percentage.

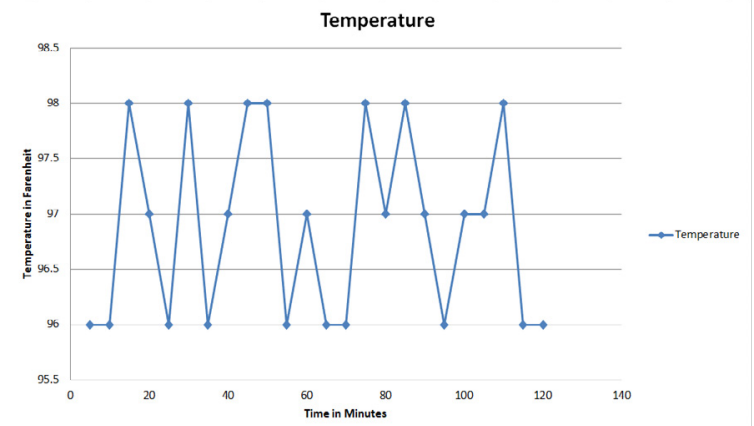

Figure 6. Graphical representation of temperature inside the incubator

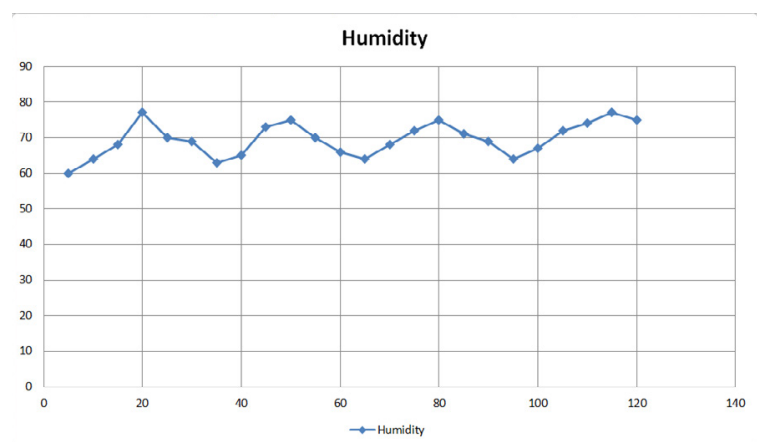

Figure 7. Graphical representation of humidity inside the incubator

\section{Discussion}

The pseudo-code of the proposed system is specified as follows:

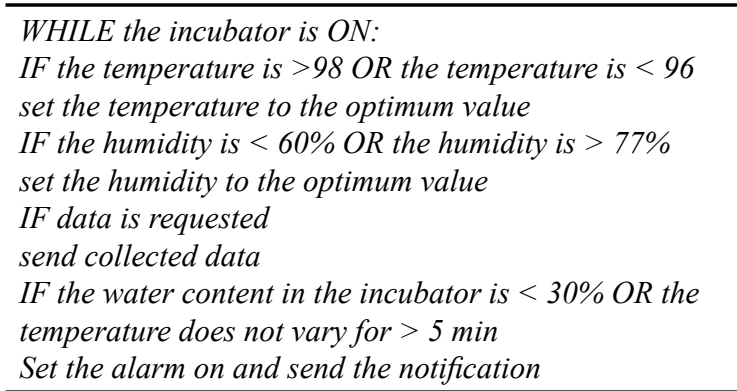

The entire system underwent a rigorous testing phase to check all the parameters of the autonomous infant incubator system. Tables 1 and 2 illustrate the various test cases applied to verify if the system operates in a specified manner. 
Table 1. Temperature description

\begin{tabular}{|c|c|c|c|c|}
\hline Test Case ID & Description & $\begin{array}{c}\text { Temperature before } \\
\text { Incubation }\end{array}$ & $\begin{array}{c}\text { Temperature during } \\
\text { Incubation }\end{array}$ & Result \\
\hline 1. & $\begin{array}{c}\text { Optimum Infant's } \\
\text { temperature }\end{array}$ & 97 & 97 & $\begin{array}{c}\text { Normal temperature } \\
\text { is maintained }\end{array}$ \\
\hline 2. & $\begin{array}{c}\text { High Infant's } \\
\text { temperature }\end{array}$ & 99 & 98 & $\begin{array}{c}\text { Temperature is decreased } \\
\text { to normal }\end{array}$ \\
\hline 3. & $\begin{array}{c}\text { Low Infant's } \\
\text { temperature }\end{array}$ & 94 & 97 & $\begin{array}{c}\text { Temperature is increased } \\
\text { to get the normal value }\end{array}$ \\
\hline 4. & $\begin{array}{c}\text { Optimum Infant's } \\
\text { temperature }\end{array}$ & 96 & 98 & $\begin{array}{c}\text { Normal temperature } \\
\text { is maintained }\end{array}$ \\
\hline 5. & $\begin{array}{c}\text { High Infant's } \\
\text { Temperature }\end{array}$ & 101 & 96 & $\begin{array}{c}\text { Temperature is decreased } \\
\text { to normal }\end{array}$ \\
\hline 6 & $\begin{array}{c}\text { Optimum Infant's } \\
\text { Temperature }\end{array}$ & 96 & 97 & $\begin{array}{c}\text { Temperature is increased } \\
\text { to get the normal value }\end{array}$ \\
\hline 7. & $\begin{array}{c}\text { Low Infant's } \\
\text { temperature }\end{array}$ & 92 & 97 temperature \\
\hline
\end{tabular}

Table 2. Humidity description

\begin{tabular}{|c|c|c|c|c|}
\hline Test Case ID & Description & $\begin{array}{c}\text { Humidity before } \\
\text { Incubation }\end{array}$ & $\begin{array}{c}\text { Humidity during } \\
\text { Incubation }\end{array}$ & Result \\
\hline 1. & Normal Infant's humidity & $65 \%$ & $65 \%$ & Normal humidity is maintained \\
\hline 2. & High Infant's humidity & $70 \%$ & $60 \%$ & $\begin{array}{c}\text { Humidity is reduced by } \\
\text { the incubator }\end{array}$ \\
\hline 3. & Low Infant's Humidity & $25 \%$ & $60 \%$ & $\begin{array}{c}\text { Humidity is increased by } \\
\text { the incubator }\end{array}$ \\
\hline 4. & Normal Infant's humidity & $65 \%$ & $65 \%$ & Normal humidity is maintained \\
\hline 5. & Low Infant's humidity & $30 \%$ & $70 \%$ & $\begin{array}{c}\text { Humidity is increased by } \\
\text { the incubator }\end{array}$ \\
\hline 6. & Optimum Infant's humidity & $63 \%$ & $64 \%$ & Normal humidity is maintained \\
\hline 7. & High Infant's humidity & $77 \%$ & $68 \%$ & Humidity is reduced by \\
the incubator
\end{tabular}

The results from Table 1 indicate that the infant's temperature should be between $96^{\circ} \mathrm{Fahrenheit}$ and $98^{\circ} \mathrm{Fahrenheit}$. If it is not the case then system fails and shows that this situation is not a normal one, then automatically sets the temperature to the optimum value, and immediately sends a notification. The humidity should be within the range of $60 \%$ to $77 \%$ as shown in Table 2 . If it is out of range, that is, becomes higher or lower, the system automatically sets the humidity to the optimum value and immediately sends a notification. This is how the incubator works.

The advantages of the proposed system are as follows:

- The smart infant incubator is built to improve the premature infant's condition and to reduce the parents' stress and make them feel calmer;

- As IBM CLOUD follows Asynchronous Request and Reply pattern the smart infant incubator's working is also based on the same pattern;

- The client information is stored in a database and is encrypted for security reasons. Information analysis techniques like quantitative, qualitative, statistical, textbased and predictive analysis are followed;

- The android app is designed to work together with the webapp by displaying the temperature and humidity values in a userfriendly manner and allowing the user to control the system;

- Many infants can be simultaneously helped in a short period of time and the incubation system keeps the infants protected, by preventing the spread of viruses and diseases to them;

- Infants in the rural areas are saved and Incubation can make infants grow faster and help them to be out of the danger zone; 
- The portability of the system combined with its low cost makes it more affordable and makes sure the ease of use;

- Secure filtering of user inputs, usage of PDO (Php Data Objects) for database operations instead of directly concatenation of user inputs. User passwords are hashed with MD5 hash, thus being harder to decrypt when the worst case of breach happens;

- System components are securely coded. Lazy coding was never followed. IBM cloud platform is already one of the most secure platforms available today. In the web module user's inputs are carefully validated and dirty inputs are filtered;

- The proposed system can prevent from Distributed Denial of Service (DDOS), SQL injection, HTML injection, XSS (Cross Site Scripting), Brute-forcing passwords, CSRF (Cross Site Request Forgery), Parameter tampering, etc.

The limitations of the proposed system are as follows:

- One of the possible limitations of the proposed work is that only the temperature and humidity parameter have been taken into consideration while disregarding other parameters like light condition and the oxygen level, up to an extent in which the preterm has the same conditions as those he had when being in his mother's womb.

- As per the lite IBM cloud plan, the smart infant incubator supports up to 800 devices.

\section{Conclusion \& Future Work}

An autonomous low-cost infant incubator using Arduino and IoT was simulated to treat premature

\section{REFERENCES}

Abdiche, M., Farges, G., Delanaud, S., Bach, V., Villon, P. \& Libert, J. P. (1998). Humidity control tool for neonatal incubator, Medical and Biological Engineering and Computing, 36(2), 241-245.

Ashish, B. (2017, February). Temperature monitored IoT based smart incubator. In 2017 International Conference on I-SMAC (IoT in Social, Mobile, Analytics and Cloud) (I-SMAC), (pp. 497-501). IEEE.

Azamir, I. M., Subramaniam, T. S. \& Fairuz, W. M. (2021). Development of Egg Incubator Monitoring System using Thingspeak as Database, Research and infants. As the success rate is high, it is greatly recommended to be used for premature infants, especially in rural areas that lack adequately equipped medical facilities, adequate maintenance, and where the cost of utilizing the incubator is high. The incubation system autonomously sets the temperature and humidity to their optimum and ideal values whey they are out of range. It also automatically notifies the doctor(s) in charge when these values are out of the range. Such a system creates an ideal environment in terms of temperature and humidity without the help of doctors or nurses. The system is secured, prevents the cyber-attacks, being resistant and resilient to these attacks, primarily because IoT is hosted on the Internet.

Secure coding practices have been used to make work less prone to anonymous attacks on the Internet. A working infant incubator system was built using the IBM IoT cloud platform. As technology advances and devices become cheaper and have better functionality, the infant incubation system could be equipped with sophisticated AI-based features and could still be offered at a low price. A future research would be to enhance the autonomous incubation system by adding an electrocardiogram (ECG) in real-time that would be sent to doctors, together with the heartbeat and pulse data. With more high-end hardware and software, the smart infant incubator can be customized, upgraded and improved for more efficiency and higher success rate. Technology is developing very fast nowadays. Costly devices are getting cheaper and cheaper devices are getting better. So infant incubator can be fitted with more advanced and sophisticated features like automated infant condition detector based on AI and can still be offered for a lower price.

Innovation in Technical and Vocational Education and Training, 1(1), 194-201.

Inam, S., Qureshi, M. F., Amin, F., Akmal, M. \& Rehman, M. Z. (2019). Android based Internet Accessible Infant Incubator. In 2019 8th International Conference on Information and Communication Technologies (ICICT), (pp. 25-29). IEEE.

Ishak, D. N. F. M., Jamil, M. A. \& Ambar, R. (2017). Arduino based infant monitoring system. In IOP Conference Series Materials Science and Engineering 226(1), (pp. 1-6). 
Jegadeesan, S., Dhamodaran, M., Azees, M. \& Shanmugapriya, S. S. (2019). Computationally efficient mutual authentication protocol for remote infant incubator monitoring system, Healthcare Technology Letters, 6(4), 92-97.

Koli, M., Ladge, P., Prasad, B., Boria, R. \& Balur, N. J. (2018, March). Intelligent baby incubator. In 2018 Second International Conference on Electronics, Communication and Aerospace Technology (ICECA), (pp. 1036-1042). IEEE.

Latif, A., Arfianto, A. Z., Poetro, J. E., Phong, T. N. \& Helmy, E. T. (2021). Temperature Monitoring System for Baby Incubator Based on Visual Basic, Journal of Robotics and Control (JRC), 2(1), 47-50.

Nisha, Z. A. \& Elahi, A. (2014). Low cost neonatal incubator with smart control system. In 8th International Conference on Software, Knowledge, Information Management \& Applications (SKIMA), Dhaka, Bangladesh, (pp. 1-5). IEEE

Rajalakshmi, A., Sunitha, K. A. \& Venkataraman, R. (2019). A survey on neonatal incubator monitoring system, Journal of Physics: Conference Series, 1362(1), 012128. DOI: 10.1088/17426596/1362/1/012128. IOP Publishing.

Rakhmawati, R., Murdianto, F. D., Luthfi, A. \& Rahman, A. Y. (2019). Thermal Optimization on Incubator using Fuzzy Inference System based IoT. In 2019 International Conference of Artificial Intelligence and Information Technology (ICAIIT), (pp. 464-468). IEEE.
Ray, P. P., Dash, D. \& Kumar, N. (2020). Sensors for Internet of medical things: State-of-the-art, security and privacy issues, challenges and future directions, Computer Communications, 160(1), 111-131.

Shabeeb, A. G., Al-Askery, A. J. \& Nahi, Z. M. (2020). Remote monitoring of a premature infant's incubator, Indonesian Journal of Electrical Engineering and Computer Science (IJEECS), 17(3), 1232-1238.

Sheet, S. S. M., Mohammed, Z. G., Khaleel, K. N., \& Abbas, A. A. (2019). Smart Infant Incubator Based on Mega Microcontroller. In 2019 2nd International Conference on Engineering Technology and its Applications (IICETA), (pp. 1-6). IEEE.

Tej, K. N., Nagabhushanam, K. \& Bachu, S. (2021). IBMPS: Incubator Baby Monitoring and Parameter Sensing. In 2021 7th International Conference on Advanced Computing and Communication Systems (ICACCS), Vol. 1 (pp. 514-518). IEEE.

Wang, W., Peng, D. \& Gu, N. (2017). Development of Monitoring System for Infant Incubator Based on IOT Technology, Chinese Journal of Medical Instrumentation, 41(3), 181-184.

Weber, R., Cabon, S., Simon, A., Porée, F. \& Carrault, G. (2021). Preterm newborn presence detection in incubator and open bed using deep transfer learning, IEEE Journal of Biomedical and Health Informatics, 25(5), 1419-1428. 